\title{
Analytical Circuit Model for 1-D Periodic T-Shaped Corrugated Surfaces
}

\author{
Carlos Molero, Raúl Rodríguez-Berral, Francisco Mesa, Fellow, IEEE, and Francisco Medina, Fellow, IEEE
}

\begin{abstract}
An analytical circuit model is obtained to study the reflection of TM polarized electromagnetic waves that impinge obliquely on a 1-D periodic corrugated surface consisting of dielectric-loaded $\mathrm{T}$-shaped planar corrugations backed by an infinite ground plane. The model is based on transmission line theory and equivalent lumped-element circuits. For the case of perfect conductors, the topology of the circuit is directly inferred from a rigorous full-wave formulation of the periodic problem without using any heuristic argument. This procedure leads to fully analytical expressions for all the circuit parameters. Ohmic losses are further incorporated in the model under the assumption of strong skin effect. The results thus obtained are compared with those given by an accurate Method of Moments numerical code and HFSS software showing a very good agreement. The strong numerical efficiency as well as the good physical insight provided by the present equivalent circuit model can be advantageously employed for the analysis and/or design of a variety of devices. As examples of the latter, the circuit model is used for the first-stage design of an electrically thin hard impedance surface, a corrugated surface that prevents specular reflection, and an absorber.
\end{abstract}

Index Terms-Corrugated surfaces, electromagnetic scattering by periodic structures, equivalent circuits.

\section{INTRODUCTION}

C ORRUGATED conducting surfaces have long been employed in microwave and antenna engineering for their unique and special electromagnetic properties. This kind of surfaces can act as guiding systems for bound surface waves and leaky waves, and their basic theory can be found in well-known textbooks [1], [2]. The control of the characteristics of these waves by proper manipulation of the geometry of the corrugations or their dielectric filling is one of the advantageous features of this type of structures. The study of these structures is a classical topic in the microwaves field and the literature on the subject can be traced to the middle decades of the past century. Thus, many authors have studied and explained the propagation

Manuscript received July 24, 2013; manuscript revised September 27, 2013; accepted November 05, 2013. Date of publication November 25, 2013; date of current version January 30, 2014. This work was supported by the Spanish Ministerio de Ciencia e Innovación and European Union FEDER funds (projects TEC2010-16948 and Consolider CSD2008-00066).

C. Molero, R. Rodríguez-Berral, and F. Mesa are with the Microwaves Group, Departamento de Física Aplicada 1, ETS de Ingeniería Informática, Universidad de Sevilla, 41012-Seville, Spain (e-mail: carmoljim@alumn.us.es; rrberral@us.es; mesa@us.es).

F. Medina is with the Departamento de Electrónica y Electromagnetismo, Facultad de Física, Universidad de Sevilla, 41012-Seville, Spain (e-mail: medina@us.es).

Color versions of one or more of the figures in this paper are available online at http://ieeexplore.ieee.org.

Digital Object Identifier 10.1109/TAP.2013.2292506

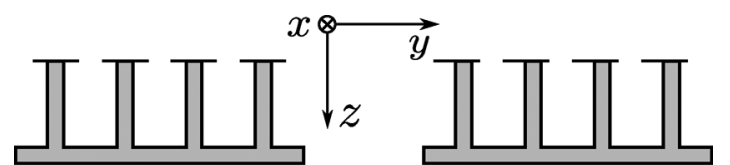

a)

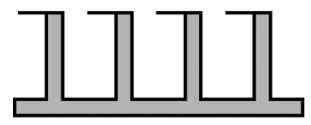

c) b)

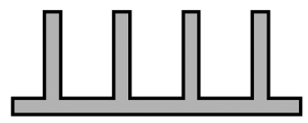

d)
Fig. 1. Cross section of the different types of corrugated planar surfaces analyzed in this work. a): T-shaped corrugated structure with a symmetrically centered slit aperture. $b$ ): T-shaped corrugated structure with a non symmetrical slit position. $c$ ): T-shaped corrugation with the maximum slit displacement. $d$ ): Classical corrugated plane. The structures are periodic along the $y$ direction and invariant along the $x$ direction.

characteristics of single planar corrugated surfaces [3]-[5], corrugated cylindrical waveguides [3], [5]-[7], or corrugated rectangular waveguides [8]. In [9], 1-D corrugated surfaces are classified as soft and hard surfaces. Accordingly, a soft corrugated surface is capable of suppressing surface waves and a hard surface enhances the wave propagation. This description is related to the value of the equivalent surface impedance of the structure, which controls the propagation properties of surface waves within the considered frequency range.

Periodically structured metal surfaces have also been considered in order to exploit their reflection/transmission characteristics as well as their focusing or field enhancement features. The interest on this kind of problems was recently boosted by the discovery of the so-called extraordinary optical transmission [10]-[12]. This phenomenon (and its dual, extraordinary reflection [11]) was originally discovered for 2-D arrays of holes in thick metal surfaces but was soon observed in 1-D periodic structures [13] similar to the ones treated in this paper. Due to this fact, many papers dealing with the analysis of metallic patterned screens can be found in physics and optics journals; see, for instance among many others, [14]-[16] or, more recently, [17]-[20] and references therein. These papers are of interest in the context of the present work because the methodology and physical discussions presented here can be applied to the problems posed in [14]-[20].

The basic geometry of the planar corrugated surfaces considered in this paper are shown in Fig. 1. It is well known that the wave guiding along a periodic structure occurs at some frequency bands (passband) and is suppressed at others (stopband) [1], [21]. Moreover, corrugated surfaces can also behave as artificial magnetic conductors (AMC), making that a plane wave impinging on the structure is reflected with near-zero phase 
shift within certain frequency band. One of the most interesting features of corrugated surfaces comes from their ability to exhibit simultaneously AMC and stopband behaviors in certain bandwidth (depending on the type of the corrugated surface, the bandwidth features can vary). This property can be efficiently used to enhance the performance of some devices, such as low-profile antennas [22]-[24]. In [25], a comparison among the stopband bandwidths obtained with the structures depicted in Fig. 1 is summarized. In general, it can be concluded that, for T-shaped corrugations, the stopband is shifted to lower frequencies as the slit is moved away from the symmetry center of the cavity. An additional bandwidth comparative study between 1-D corrugated surfaces and the well-known 2-D mushroom configurations [22] is also given in [25].

The structures in Fig. 1 were rigorously analyzed using the aperture integral equation combined with the method of moments in [26]. In a more recent paper [27], the symmetric T-shaped corrugated structure shown in Fig. 1(a) is analyzed using both numerical and analytical approaches. Both AMC bandwidth and surface-wave stopband are studied in [27], and a simple formula (with certain parameters to be fitted) is derived using the transverse resonant technique to calculate the phase of the reflection coefficient when the structure is illuminated by an obliquely incident plane wave. This formula seems to work properly for narrow corrugations but it will be shown here that it fails in predicting the behavior of the structure if the groove size is similar to the period. An enhanced theory is presented in [28] for the case of planar corrugated planes, but it does not consider T-shaped corrugations, limiting the range of applicability to the classical configuration shown in Fig. 1(d). For this latter configuration, another circuit model is presented in [29] to compute the reflection coefficient of the structure under oblique incidence.

In order to extend the possibility of using closed-form analytical formulas to all the configurations shown in Fig. 1, this paper presents a novel circuit model to obtain the reflection properties of a TM oblique plane wave impinging on those corrugated surfaces. (The case of TE polarization is not explicitly treated here since the interaction with the structure is very weak in such case. However, the analysis of this case can be carried out along very similar lines). The present analysis is based on previous ideas and works developed in [30], [31] and, especially, [32], where a circuit model was systematically deduced for the scattering problem in slits/strips gratings embedded in a layered environment. The whole formulation is described in next section for a general case that considers the corrugations filled with a dielectric material, the presence of a dielectric overlay on top of them, and ohmic losses in the cavity walls. Different comparisons with a self-developed Method of Moments (MoM) code and with ANSYS HFSS [33] are presented, as well as with previously reported models [27], [29]. Finally, our circuit approach is employed to propose examples of some possible applications of this type of structures.

\section{Derivation of the Analytical Model}

The T-shaped corrugated structures under consideration in this work are those shown in Fig. 1 but including the possible presence of dielectric filling and cover. The whole structure

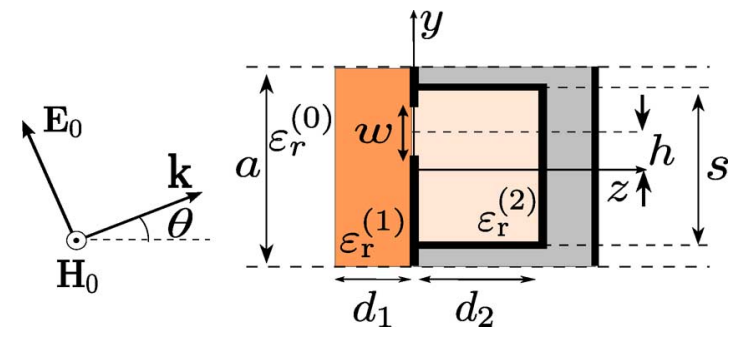

Fig. 2. General unit cell of any of the structures shown in Fig. 1 including a cover slab and a cavity dielectric filling. Structural parameters: period $a$, groove size $s$, slit width $w$, slit displacement $h$, external slab thickness $d_{1}$, corrugation depth $d_{2}$, relative permittivity of the dielectric in the cavity $\varepsilon_{\mathrm{r}}^{(2)}$, relative permittivity in the external slab $\varepsilon_{\mathrm{r}}^{(1)}$, external relative permittivity $\varepsilon_{\mathrm{r}}^{(0)}$, incidence angle $\theta$.

extends indefinitely along the $x$ direction, which makes the structure be 1-D periodic along the $y$-direction. A TM-polarized plane wave impinges on the structure, exciting electric currents on the metallic surfaces. Due to the periodic nature of the structure, Floquet theory allows us to restrict the analysis to the unit cell of period $a$ shown in Fig. 2 (it should be noted that all the configurations sketched in Fig. 1 are particular cases of the generalized unit cell defined in Fig. 2).

\section{A. Lossless Case}

The unit cell shown in Fig. 2 involves two clearly distinct regions: (1) the external region $(z<0)$, which is regarded as a generalized parallel-plate waveguide with periodic boundary conditions at its upper and lower walls (a dielectric overlay can eventually be placed in the external region above the corrugations at $-d_{1}<z<0$ ), and (2) the cavity region, a parallel-plate waveguide with electric-wall boundary conditions and short-circuited at its right end $\left(z=d_{2}\right)$. Employing a Floquet expansion to describe the electric field in the external region (1) and a modal expansion in the parallel-plate waveguide corresponding to the cavity region (2), the $y$-component of the electric field at both sides of the $z=0$ plane can be written as

$$
\begin{aligned}
& E^{(1)}(y)=\left[1+R^{(1)}+\sum_{\forall n \neq 0} E_{n}^{(1)} \mathrm{e}^{-\mathrm{j} k_{n}^{(1)} y}\right] \mathrm{e}^{-\mathrm{j} k_{t} y} \\
& E^{(2)}(y)=\sum_{n=0}^{\infty} E_{2 n}^{(2)} \cos \left(k_{2 n}^{(2)} y\right)+\sum_{n=0}^{\infty} E_{2 n+1}^{(2)} \sin \left(k_{2 n+1}^{(2)} y\right)
\end{aligned}
$$

The cutoff wavenumber parameters are $k_{n}^{(1)}=2 \pi n / a$ and $k_{n}^{(2)}=\pi n / s$. The amplitude of the impinging plane wave has been normalized to unity, $R^{(1)}$ represents the reflection coefficient of the reflected plane wave inside the dielectric overlay, and $k_{t}=\left[\varepsilon_{\mathrm{r}}^{(0)}\right]^{1 / 2} k_{0} \sin \theta$ is the tangential (to the grating) component of the wavevector of the obliquely incident plane wave, with $k_{0}=\omega / c$ being the free-space wavenumber ( $\omega$ is the angular frequency of the incident time-harmonic field and $c$ the speed of light in free space) and $\theta$ the incidence angle.

At this point, a key assumption is made that the electric field at the slit aperture can be factorized in the following way [32]:

$$
E_{\mathrm{a}}(\omega, y)=A(\omega) f(y)=A(\omega) \frac{\frac{2}{\pi}}{\sqrt{1-\left(\frac{2(y-h)}{w}\right)^{2}}} .
$$


The spatial field profile, $f(y)$, incorporates the exact edge singularities when the edges of the slit are not placed at $y= \pm s / 2$ (see Fig. 2). In any case, it is expected to be a good approximation for apertures whose width is electrically narrow. Projecting each harmonic/mode over $f(y)$ and using Fourier analysis, the following coefficients of the field expansion are obtained:

$$
\begin{aligned}
1+R^{(1)} & =\frac{A(\omega)}{a} J_{0}\left(k_{t} \frac{w}{2}\right) \mathrm{e}^{\mathrm{j} k_{t} h} \\
E_{n}^{(1)} & =\frac{A(\omega)}{a} J_{0}\left(\left(k_{t}+k_{n}^{(1)}\right) \frac{w}{2}\right) \mathrm{e}^{\mathrm{j}\left(k_{t}+k_{n}^{(1)}\right) h} \\
E_{0}^{(2)} & =\frac{A(\omega)}{s} \\
E_{2 n}^{(2)} & =\frac{2 A(\omega)}{s} J_{0}\left(k_{2 n}^{(2)} \frac{w}{2}\right) \cos \left(k_{2 n}^{(2)} h\right) \\
E_{2 n+1}^{(2)} & =\frac{2 A(\omega)}{s} J_{0}\left(k_{2 n+1}^{(2)} \frac{w}{2}\right) \sin \left(k_{2 n+1}^{(2)} h\right)
\end{aligned}
$$

where $J_{0}(\cdot)$ is the zero-order Bessel function of the first kind. From the above equations, all the coefficients can be written in terms of $R^{(1)}$ as

$$
\begin{aligned}
E_{n}^{(1)} & =\left(1+R^{(1)}\right) \frac{J_{0}\left(\left(k_{t}+k_{n}^{(1)}\right) \frac{w}{2}\right)}{J_{0}\left(k_{t} \frac{w}{2}\right)} \mathrm{e}^{\mathrm{j} k_{n}^{(1)} h} \\
E_{2 n}^{(2)} & =\left(2-\delta_{n, 0}\right)\left(1+R^{(1)}\right) \frac{a}{s} \frac{J_{0}\left(k_{2 n}^{(2)} \frac{w}{2}\right)}{J_{0}\left(k_{t} \frac{w}{2}\right) \mathrm{e}^{\mathrm{j} k_{t} h}} \cos \left(k_{2 n}^{(2)} h\right) \\
E_{2 n+1}^{(2)} & =2\left(1+R^{(1)}\right) \frac{a}{s} \frac{J_{0}\left(k_{2 n+1}^{(2)} \frac{w}{2}\right)}{J_{0}\left(k_{t} \frac{w}{2}\right) \mathrm{e}^{\mathrm{j} k_{t} h}} \sin \left(k_{2 n+1}^{(2)} h\right)
\end{aligned}
$$

Following a similar procedure as in [32], the continuity of the magnetic field ( $x$-component) through the slit

$$
H^{(1)}(y)=H^{(2)}(y) \quad, \quad h-\frac{w}{2}<y<h+\frac{w}{2}
$$

can now be written in terms of the coefficients of the modal expansions of the electric field in the following way:

$$
\begin{aligned}
{\left[\left(1-R^{(1)}\right) Y_{0}^{(1)}\right.} & \left.-\sum_{\forall n \neq 0} Y_{n}^{\mathrm{in},(1)} E_{n}^{(1)} \mathrm{e}^{-\mathrm{j} k_{n}^{(1)} y}\right] \mathrm{e}^{-\mathrm{j} k_{t} y} \\
= & \sum_{n=0}^{\infty} Y_{2 n}^{\mathrm{in},(2)} E_{2 n}^{(2)} \cos \left(k_{2 n}^{(2)} y\right) \\
& +\sum_{n=0}^{\infty} Y_{2 n+1}^{\mathrm{in},(2)} E_{2 n+1}^{(2)} \sin \left(k_{2 n+1}^{(2)} y\right)
\end{aligned}
$$

where

$$
\begin{aligned}
& Y_{n}^{\mathrm{in},(1)}=Y_{n}^{(1)} \frac{Y_{n}^{(0)}+\mathrm{j} Y_{n}^{(1)} \tan \left(\beta_{n}^{(1)} d_{1}\right)}{Y_{n}^{(1)}+\mathrm{j} Y_{n}^{(0)} \tan \left(\beta_{n}^{(1)} d_{1}\right)} \\
& Y_{n}^{\mathrm{in},(2)}=-\mathrm{j} Y_{n}^{(2)} \cot \left(\beta_{n}^{(2)} d_{2}\right)
\end{aligned}
$$

can be interpreted as the input admittances corresponding to the transmission lines associated with the high order modes/ harmonics excited at both sides of the discontinuity, with

$$
Y_{n}^{(i)}=\frac{\varepsilon_{\mathrm{r}}^{(i)} k_{0}}{\eta_{0} \beta_{n}^{(i)}} \quad(i=0,1,2)
$$

being the characteristic admittance $\left(\eta_{0}\right.$ is the free space impedance) of the transmission line associated with the $n$-th mode/harmonic in the $i$-th medium, and

$$
\begin{aligned}
& \beta_{n}^{(i)}=\sqrt{\varepsilon_{\mathrm{r}}^{(i)} k_{0}^{2}-\left[k_{t}+k_{n}^{(1)}\right]^{2}} \quad(i=0,1) \\
& \beta_{n}^{(2)}=\sqrt{\varepsilon_{\mathrm{r}}^{(2)} k_{0}^{2}-\left[k_{n}^{(2)}\right]^{2}}
\end{aligned}
$$

the corresponding modal/harmonic wavenumbers. After projecting (13) over the profile function

$$
\int_{h-w / 2}^{h+w / 2} f(y)\left[H^{(1)}(y)-H^{(2)}(y)\right] \mathrm{d} y=0
$$

and employing (9), (10) and (11), the following expression for the reflection coefficient of the impinging plane wave is found:

$$
R^{(1)}=\frac{Y_{0}^{(1)}-A_{0}^{(2)} Y_{0}^{\mathrm{in},(2)}-Y_{\mathrm{eq}}}{Y_{0}^{(1)}+A_{0}^{(2)} Y_{0}^{\mathrm{in},(2)}+Y_{\mathrm{eq}}}
$$

where the equivalent admittance of the discontinuity is given by $Y_{\mathrm{eq}}=\sum_{|n|=1}^{\infty} A_{n}^{(1)} Y_{n}^{\mathrm{in},(1)}+\sum_{n=1}^{\infty} A_{2 n}^{(2)} Y_{2 n}^{\mathrm{in},(2)}+\sum_{n=0}^{\infty} A_{2 n+1}^{(2)} Y_{2 n+1}^{\mathrm{in},(2)}$

with

$$
\begin{aligned}
A_{n}^{(1)} & =\frac{J_{0}^{2}\left(\left(k_{t}+k_{n}^{(1)}\right) \frac{w}{2}\right)}{J_{0}^{2}\left(k_{t} \frac{w}{2}\right)} \\
A_{2 n}^{(2)} & =\left(2-\delta_{n, 0}\right) \frac{a}{s} \frac{J_{0}^{2}\left(k_{2 n}^{(2)} \frac{w}{2}\right)}{J_{0}^{2}\left(k_{t} \frac{w}{2}\right)} \cos ^{2}\left(k_{2 n}^{(2)} h\right) \\
A_{2 n+1}^{(2)} & =\frac{2 a}{s} \frac{J_{0}^{2}\left(k_{2 n+1}^{(2)} \frac{w}{2}\right)}{J_{0}^{2}\left(k_{t} \frac{w}{2}\right)} \sin ^{2}\left(k_{2 n+1}^{(2)} h\right) .
\end{aligned}
$$

The expression (20) obtained above for the reflection coefficient, together with the interpretation of the input admittances, leads us to the equivalent circuit depicted in Fig. 3(a). Concerning the topology of the $Y_{\text {eq }}$ admittance in the circuit model, from (21) it is apparent that it consists of a parallel connection of the input admittances of all the high order harmonics (open region) and modes (cavity region) excited at the discontinuity (weighted by the factor $A_{n}^{(i)}$ ). The $Y_{\text {eq }}$ is thus formally given by the parallel connection of an infinite number of input admittances. This infinite series is not very convenient from a practical point of view, but this drawback can be overcome following a strategy similar to that in [32]. The basic idea consists in dealing separately with the overall contribution of all the high order harmonics/modes that are far from their cutoff frequencies. For harmonics and modes with high cutoff wavenumbers (in comparison to $\left[\varepsilon_{\mathrm{r}}^{(i)}\right]^{1 / 2} k_{0}$ ), the following approximations are used:

$$
\beta_{n}^{(i)} \approx-\mathrm{j} k_{|n|}^{(i)}, \quad k_{t}+k_{n}^{(1)} \approx k_{n}^{(1)}
$$

and their characteristic admittances reduce to

$$
Y_{n}^{(i)} \approx \mathrm{j} \omega \frac{\varepsilon_{0} \varepsilon_{\mathrm{r}}^{(i)}}{k_{n}^{(i)}}=\mathrm{j} \omega C_{n}^{(i)} .
$$




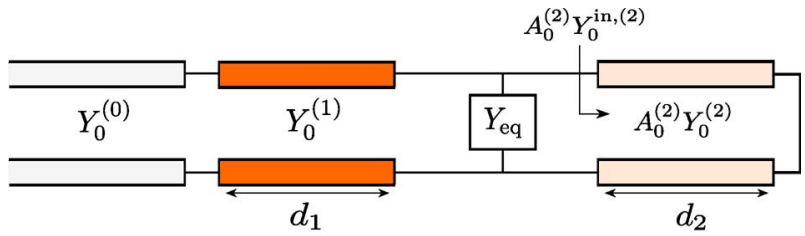

(a)

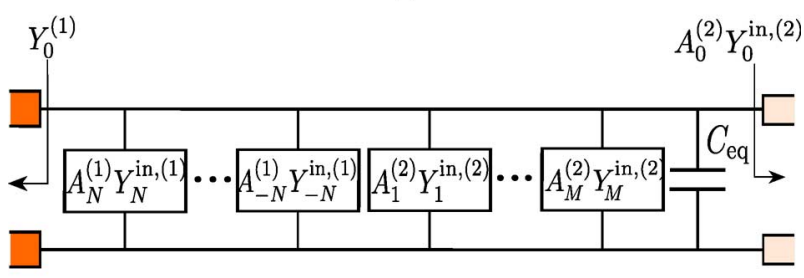

(b)
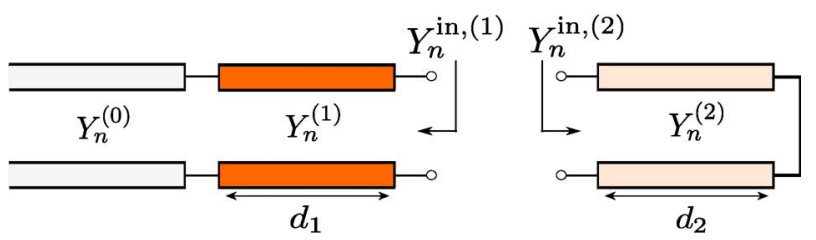

(c)

Fig. 3. (a) Equivalent circuit of the periodic structure under analysis. (b) Topology of the equivalent admittance $Y_{\text {eq }}$ that represents the high-order harmonics and modes excited at the slit discontinuity. (c) Definition of the input admittances in $Y_{\text {eq }}$.

In this way, all the parallel-connected admittances of high order (greater than, say, a given order $N$ for the external region and $M$ for the cavity) can be grouped together to form an equivalent high order capacitance, $C_{\text {eq }}$, given by

$$
\begin{aligned}
C_{\mathrm{eq}} & =\frac{2}{J_{0}^{2}\left(k_{t} \frac{w}{2}\right)} \\
& \times\left\{\sum_{n=N+1}^{\infty} J_{0}^{2}\left(k_{n}^{(1)} \frac{w}{2}\right) \frac{\varepsilon_{\mathrm{r}}^{(0)}+\varepsilon_{\mathrm{r}}^{(1)} \tanh \left(k_{n}^{(1)} d_{1}\right)}{\varepsilon_{\mathrm{r}}^{(1)}+\varepsilon_{\mathrm{r}}^{(0)} \tanh \left(k_{n}^{(1)} d_{1}\right)} C_{n}^{(1)}\right. \\
& +\sum_{n=M+1}^{\infty}\left[\frac{a}{s} J_{0}^{2}\left(k_{2 n}^{(2)} \frac{w}{2}\right) \cos ^{2}\left(k_{2 n}^{(2)} h\right) \operatorname{coth}\left(k_{2 n}^{(2)} d_{2}\right) C_{2 n}^{(2)}\right. \\
& \left.\left.+\frac{a}{s} J_{0}^{2}\left(k_{2 n+1}^{(2)} \frac{w}{2}\right) \sin ^{2}\left(k_{2 n+1}^{(2)} h\right) \operatorname{coth}\left(k_{2 n+1}^{(2)} d_{2}\right) C_{2 n+1}^{(2)}\right]\right\} .
\end{aligned}
$$

Thus, the equivalent admittance can finally be written as a sum of a few dynamic terms plus the contribution of the equivalent high-order capacitance:

$$
\begin{aligned}
Y_{\mathrm{eq}}=\sum_{|n|=1}^{N} A_{n}^{(1)} Y_{n}^{\mathrm{in},(1)} & +\sum_{n=1}^{M} A_{2 n}^{(2)} Y_{2 n}^{\mathrm{in},(2)} \\
& +\sum_{n=0}^{M} A_{2 n+1}^{(2)} Y_{2 n+1}^{\mathrm{in},(2)}+\mathrm{j} \omega C_{\mathrm{eq}} .
\end{aligned}
$$

Taking into account this last expression, the topology of the equivalent admittance $Y_{\text {eq }}$ is shown in Fig. 3(b). It should be noted that the infinite series in the capacitance $C_{\text {eq }}$ [see (27)] is independent of both frequency and incidence angle. Therefore, in the case of an eventual sweep in either frequency or incidence angle, the infinite series needs to be computed only once [32]. Concerning the value of $N$ and $M$, in practice they can be chosen as the order of the first harmonic/mode that remains evanescent in their respective medium at the highest frequency of interest [32] (in most practical cases, they are rarely higher than three). The low order elements in (28) would then account analytically for the nontrivial frequency dependence of the equivalent admittance of the first relevant high order harmonics/modes. It is interesting to note that the value of the groove depth $\left(d_{2}\right)$ is not relevant in the choice of $M$ ( $M$ is only determined by $\left.\beta_{n}^{(2)} \approx-\mathrm{j} k_{|n|}^{(2)}\right)$. But this does not mean that for shallow grooves the coupling of the slit aperture with the back metallic wall through high-order evanescent modes is being somehow neglected. Indeed, this coupling is accurately taken into account by the $\operatorname{coth}\left(k_{n}^{(2)} d_{2}\right)$ factors in (27), whose value is different from one for evanescent modes that reach the back wall at $z=d_{2}$.

Finally, it should be recalled that $R^{(1)}$ above represents the reflection coefficient seen at $z=0$. The $S_{11}$ parameter of the incident plane wave at $z=-d_{1}$ is then given by

$$
S_{11}=\frac{\left(1-\frac{Y_{0}^{(1)}}{Y_{0}^{(0)}}\right)+\left(1+\frac{Y_{0}^{(1)}}{Y_{0}^{(0)}}\right) R^{(1)} \mathrm{e}^{-2 \mathrm{j} \beta_{0}^{(1)} d_{1}}}{\left(1+\frac{Y_{0}^{(1)}}{Y_{0}^{(0)}}\right)+\left(1-\frac{Y_{0}^{(1)}}{Y_{0}^{(0)}}\right) R^{(1)} \mathrm{e}^{-2 \mathrm{j} \beta_{0}^{(1)} d_{1}}} .
$$

It should be pointed out that the procedure described in this section is extremely fast from a computational point of view. All the computations involved in the study of the cases considered in Section III of this work have taken an almost negligible CPU time in a standard laptop computer when compared with the use of numerical methods.

\section{B. Resistive Overlay}

If the dielectric overlay [medium (1)] is lossy (this is an interesting situation if the structure is intended to be used as absorber), its permittivity is complex and can be written as $\hat{\varepsilon}^{(1)}=\varepsilon_{0} \hat{\varepsilon}_{\mathbf{r}}^{(1)}$ with

$$
\hat{\varepsilon}_{\mathrm{r}}^{(1)}=\varepsilon_{\mathrm{r}}^{(1)}(1-\mathrm{j} \tan \delta)-\mathrm{j} \frac{\sigma_{1}}{\omega \varepsilon_{0}}
$$

where $\delta$ represents the dielectric loss tangent and $\sigma_{1}$ the conductivity of the medium. The complex wavenumber of the $n$-th harmonic in this medium is then given by

$$
\hat{\beta}_{n}^{(1)}=\sqrt{\hat{\varepsilon}_{\mathrm{r}}^{(1)} k_{0}^{2}-\left(k_{n}^{(1)}+k_{t}\right)^{2}} .
$$

The complex values of the relative permittivity and the wavenumber given in (30) and (31) must now be introduced in (16) to obtain the associated complex harmonic admittance, $\hat{Y}_{n}^{(1)}$, in this lossy medium. (A completely similar treatment applies in case the dielectric filling the cavity is lossy.)

\section{Ohmic Losses in the Cavity Walls}

For completeness, ohmic losses in the cavity walls are also incorporated in the model. Since the metallic materials usually employed in the fabrication of practical structures are good conductors, the conventional strong skin-effect approximation is used here. The effect of losses in the lateral walls at $y= \pm s / 2$, 
the back wall at $z=d_{2}$, and the periodic slit screen are considered separately. In any case, an additional approximation is made that conductor ohmic losses are attributed only to propagative modes.

Ohmic losses in the lateral walls cause the wavenumbers of the parallel plate waveguide modes to become complex. Their quantitative impact is obtained via the well-known perturbation method [2], which provides not only a resistive but also an inductive contribution due to the penetration of the magnetic field into the metal (the inductive contribution is usually negligible, but may become noticeable for very high frequencies and/or very narrow cavities). Thus, the complex wavenumber of each modal transmission line in the cavity can now be written as

$$
\hat{\beta}_{n}^{(2)}=\sqrt{\left(\beta_{n}^{(2)}\right)^{2}+\left(\Delta \beta_{n}\right)^{2}}
$$

where $\beta_{n}^{(2)}$ is the propagation constant calculated in the lossless case [see (18)], and $\left(\Delta \beta_{n}\right)^{2}$ is the contribution of the ohmic losses obtained by the perturbation method

$$
\left(\Delta \beta_{n}\right)^{2}=\left(2-\delta_{n, 0}\right)(1-\mathrm{j}) \frac{\delta_{s}}{s} \varepsilon_{\mathrm{r}}^{(2)} k_{0}^{2}
$$

with $\delta_{s}=\sqrt{2 / \omega \mu_{0} \sigma_{\mathrm{m}}}$ being the skin depth and $\sigma_{\mathrm{m}}$ the conductivity of the metal. The complex characteristic admittance of the corresponding $n$-order transmission line, $\hat{Y}_{n}^{(2)}$, is obtained after introducing (32) into (16).

Concerning ohmic losses in the back wall of the cavity, its effect is introduced by placing an imperfect short-circuit load at the end of the modal transmission lines. Assuming strong skin effect, the value of the admittance of this load is given directly by the surface admittance [2]

$$
Y_{s}=\frac{1}{2}(1-\mathrm{j}) \sigma_{\mathrm{m}} \delta_{s}
$$

which is the same for all the cavity modes.

For the two previous cases of lateral walls and back wall it has been possible to account approximately for the effect of ohmic losses individually for each mode in the cavity, and then it is straightforward to compute the corresponding input admittances seen at the slit aperture. These input admittances incorporate the combined effect of the lateral and back wall losses and are obtained by transforming the imperfect load $Y_{s}$ along the lossy transmission line with wavenumber $\hat{\beta}_{n}^{(2)}$ and characteristic admittance $\hat{Y}_{n}^{(2)}$, thus giving the following expression that substitutes $(15)$ :

$$
\hat{Y}_{\mathrm{in}, n}^{(2)}=\hat{Y}_{n}^{(2)} \frac{Y_{s}+\mathrm{j} \hat{Y}_{n}^{(2)} \tan \left(\hat{\beta}_{n}^{(2)} d_{2}\right)}{\hat{Y}_{n}^{(2)}+\mathrm{j} Y_{s} \tan \left(\hat{\beta}_{n}^{(2)} d_{2}\right)} .
$$

Finally, for ohmic losses in the slit screen, since all modes and harmonics couple together at the slit discontinuity, it is not easy to find an approximate analytical expression that accounts for the effect of each individual mode. However, a heuristic reasoning suggests to model the resistive screen by a similar equivalent surface admittance as that used in the back wall case, but excluding now the contribution corresponding to the aperture. Thus, this ohmic effect is here taken into account as a se-

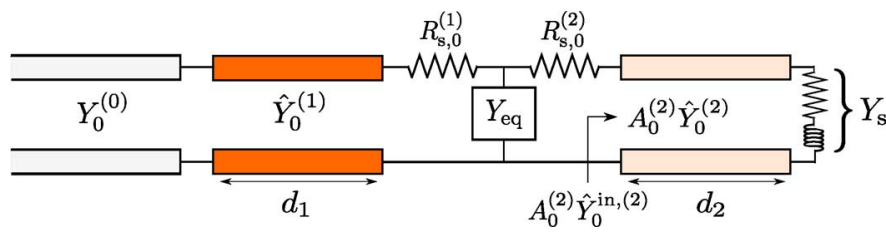

Fig. 4. Equivalent circuit of the periodic structure under analysis with ohmic losses.

ries resistance connected to each transmission line (propagative modes/harmonics), whose value is approximated as

$$
\begin{aligned}
& R_{s, n}^{(1)}=\frac{a-w}{a} \frac{1}{\sigma_{\mathrm{m}} \delta_{s}} \\
& R_{s, n}^{(2)}=2\left(1-\delta_{n, 0}\right) \frac{a}{s} \frac{s-w}{s} \frac{1}{\sigma_{\mathrm{m}} \delta_{s}} .
\end{aligned}
$$

The resulting final equivalent circuit is schematically shown in Fig. 4.

\section{NUMERICAL RESULTS}

In the derivation of our equivalent circuit model, it was assumed a given electric field profile at the slit aperture. For the strip/slit gratings studied in [32], this approximated profile was proven to give sufficient accuracy, even in the oblique incidence case. This fact suggests that the same profile could also work properly for both the symmetric and the nonsymmetric planar T-shaped corrugated structures. In this latter case, the asymmetry is approximately considered in our analysis by the inclusion of odd modes in the proposed modal expansion for the cavity. An additional approximation of our study is that the effect of higher order harmonics/modes is accounted for by a global lumped capacitance. Hence, due to the existence of such approximations, the accuracy of our model needs to be validated against some independent numerical results. This validation is next carried out by comparing our results with the data obtained from a full-wave MoM numerical code that uses several basis functions for the electric field at the slit (for our purposes, this carefully implemented MoM is considered "exact"). With the aim of exploring how other previously proposed equivalent-circuit models behave with respect to our equivalent-circuit approach in terms of accuracy, robustness and range of applicability, our results are also compared with those computed from the formula provided in [27, Eq.27] (henceforth called Kehn's formula) and with those obtained following the Woo's circuit reported in [29]. The examples showing ohmic losses are compared with simulations obtained with HFSS.

A first comparison is shown in Fig. 5, where the phase of the reflection coefficient is plotted versus frequency for three different configurations (see the caption for the structure parameters). The slit aperture is symmetrically placed, so there is no contribution of the odd modes inside the cavity in our model. As expected, as frequency increases, the structure departs from the low-frequency electric wall behavior to achieve a near-zero phase of the reflection coefficient (artificial magnetic conductor or high-impedance surface) in a given frequency range. This can be understood as the "quarter-wave transformer" effect caused by the transmission line corresponding to the fundamental TEM mode inside the groove 

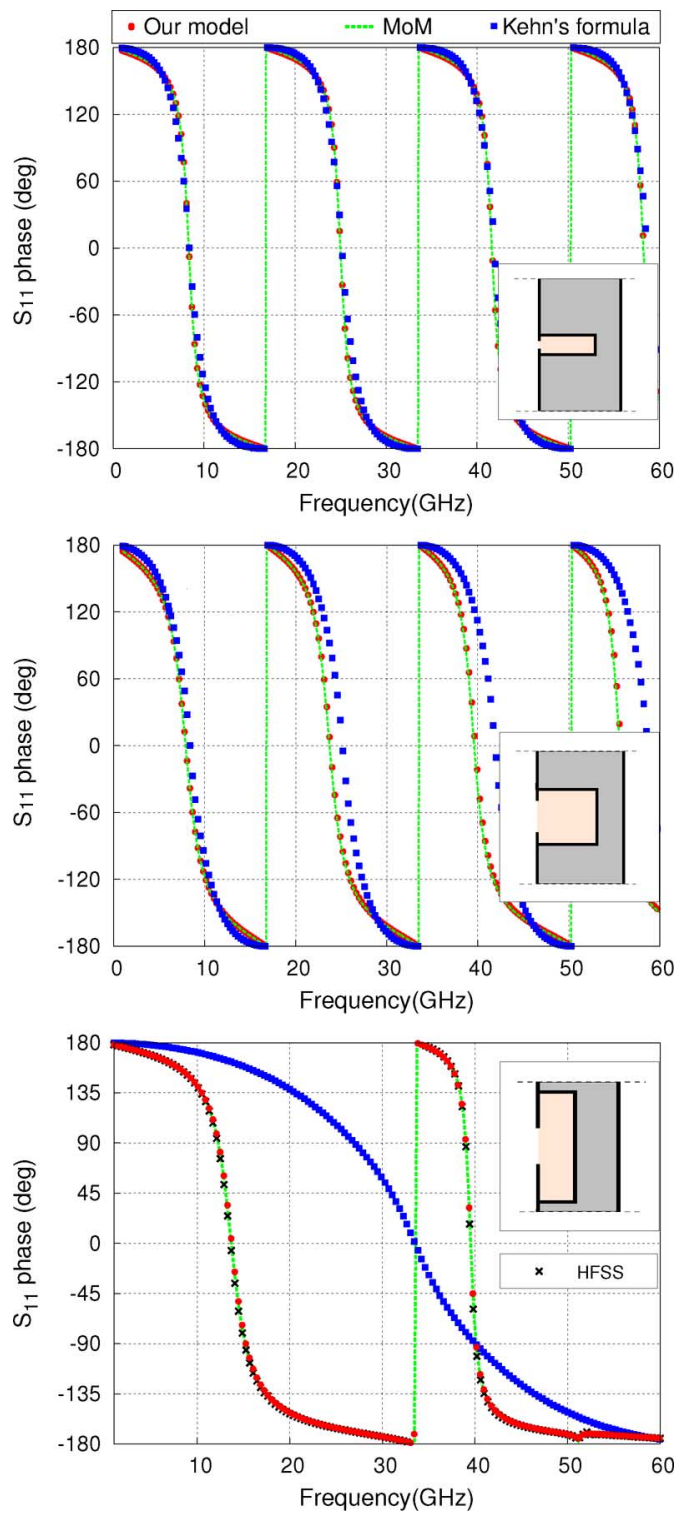

Fig. 5. Phase of the reflection coefficient versus frequency for three different configurations. Top: $a=1 \mathrm{~mm}, s=0.25 \mathrm{~mm}, w=0.75 \mathrm{~s}, d_{2}=4 \mathrm{~mm}$, $h=0 \mathrm{~mm}, \varepsilon_{\mathrm{r}}^{(1)}=1, \varepsilon_{\mathrm{r}}^{(2)}=5, \theta=30^{\circ}$, in our model $N=2, M=1$. Center: Same as top, except $s=0.5 \mathrm{~mm}, w=0.2 \mathrm{~s}$. Bottom: $a=5 \mathrm{~mm}$, $s=4 \mathrm{~mm}, w=0.2 \mathrm{~s}, d_{2}=1 \mathrm{~mm}, h=0 \mathrm{~mm}, \varepsilon_{\mathrm{r}}^{(1)}=1, \varepsilon_{\mathrm{r}}^{(2)}=5, \theta=10^{\circ}$. In our model, $N=2, M=2$.

[the transmission line (2) shown in Fig. 3(a)]. Rigorously, the magnetic wall condition occurs at a frequency slightly lower than that for which $Y_{0}^{\text {in, }(2)}=0$, since this admittance has to compensate for the capacitive admittance $Y_{\mathrm{eq}}$ introduced by the high-order modes/harmonics excited at the slit discontinuity so that $Y_{0}^{\mathrm{in},(2)}+Y_{\mathrm{eq}}=0$. In the case shown at the top of Fig. 5, the agreement between the three sets of data (our equivalent circuit, the MoM, and Kehn's formula in [27] and [28]) is very good in all the frequency range. However, in this case, the groove width is electrically small in the considered frequency range ( $s / \lambda_{0} \leq 1 / 20$, with $\lambda_{0}$ being the free-space wavelength) and the slit aperture is relatively wide with respect to the groove $(w / s=3 / 4)$. Hence, the high-order modes excited at the discontinuity are not expected to have a relevant effect (Khen's formula was derived neglecting the effect of high order modes). In the case shown at the central panel of Fig. 5, the groove has been enlarged to half the size of the unit-cell period $(s / a=1 / 2)$, and the slit aperture has been narrowed to $20 \%$ of the groove size $(w / s=1 / 5)$. In this situation, Kehn's formula clearly deviates from the MoM numerical results as frequency increases, whereas the results provided by our circuit model remain remarkably accurate. With the set of dimensions chosen in the bottom panel in Fig. 5, Kehn's formula is not capable of reproducing the correct behavior of the phase of the reflection coefficient. The reason behind such a poor behavior of Kehn's expression is related to the relatively small value of the corrugation depth. Under these circumstances, the implicit assumption in Kehn's model that links the phase evolution to the existence of Fabry-Pérot resonances along the $z$-direction is no longer valid. Our model makes no assumption in this regard but takes into account analytically the influence of high order cavity modes, which turns out to be essential in this situation. Indeed, the cutoff frequency of the first high order mode inside the cavity is $33.54 \mathrm{GHz}$. At this frequency, the admittance of this high order mode diverges, giving rise to a short-circuit in the equivalent admittance associated with the discontinuity. This fact is perfectly captured by our model, in good agreement with MoM, but seems to be ignored by other simplified equivalent circuits. (In order to show a validation of our MoM code, HFSS results are also included in this last plot. The agreement between MoM and HFSS results is similarly good for all the cases without ohmic losses studied.)

Fig. 6 shows results for the classical corrugated surface recently studied in [29] by means of an analytical circuit model (here called Woo's circuit model). Actually, for the case of TM incidence, the circuit model in [29] resembles the one proposed by some of the authors in [34], [35] for transmission slit structures. The purpose of Fig. 6 is to compare the predictions of the model in [29] with the new proposal in the present paper. The top plot shows the phase of the reflection coefficient when the groove size is $1 / 10$ of the unit cell period. As expected, our circuit approach gives accurate results, which agree very well with MoM data, due to the small electrical size of the aperture. Woo's circuit model also provides acceptable agreement with MoM numerical code results, although slightly less precise. It should be reminded that Woo's model considers only the TEM mode inside the cavity; i.e., the reactive field is ignored. Hence, as the groove size grows, the accuracy of this model is expected to worsen, as observed in the bottom plot of Fig. 6 even in the low frequency regime. In this last case, the groove size is now $4 / 10$ of the period and its electrical size is not negligible at the high end of the studied frequency spectrum, thus inducing a non-negligible reactive field inside the cavity. Although our model does account for the reactive field excited in the discontinuity, its numerical results are not expected to be very accurate at high frequencies where the width of the cavity is not electrically small (@45 GHz, $\left.s / \lambda_{0}=3 / 10\right)$ and the approximation for the aperture field in (3) is not sufficiently valid.

An interesting example is shown in Fig. 7, where the magnitude and phase of the reflection coefficient is plotted versus frequency for a nonsymmetric T-shaped corrugated structure. Since an obliquely incident plane wave impinges on the corrugated surface, purely specular reflection is expected only below approximately $34 \mathrm{GHz}$, the onset frequency at which the $n=-1$ 

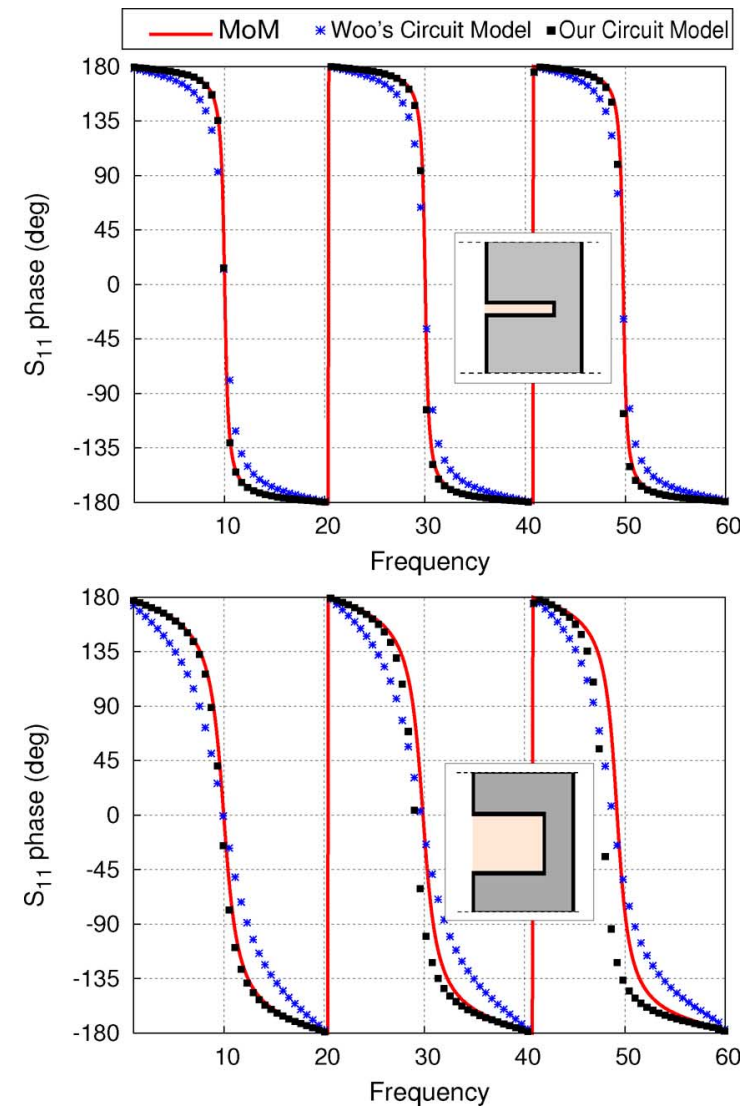

Fig. 6. Phase of reflection coefficient versus frequency for two different configurations. Top: $a=5 \mathrm{~mm}, s=0.5 \mathrm{~mm}, w=s, d=3 \mathrm{~mm}, h=0 \mathrm{~mm}$, $\varepsilon_{\mathrm{r}}^{(1)}=1, \varepsilon_{\mathrm{r}}^{(2)}=6, \theta=0^{\circ}$, in our model $N=1, M=1$. Bottom: Same as top, except $s=2 \mathrm{~mm}$ and $M=2$.

harmonic becomes propagative. Above that onset frequency, the reflected power is carried away by the two propagative harmonics $(n=0,-1)$ outside the cavity. As frequency increases, the power ratio transferred to each propagative harmonic varies. The top plot in Fig. 7 shows that, around 41.2 and $52.7 \mathrm{GHz}$, the fraction of the reflected power channeled into the zero-order harmonic is very low. In certain frequency bands centered at these two frequencies, most of the power is then "diffracted" to the $n=-1$ harmonic, thus preventing specular reflection. This behavior can readily be understood in terms of our circuit model, as explained next. In the bottom plot of Fig. 7, it can be observed that the reflection phase is close to zero at these two reflectivity minima. Indeed, the minima occur when the corrugation depth $d_{2}$ is below but close to $7 / 4 \lambda_{0}^{(2)}$ and $9 / 4 \lambda_{0}^{(2)}$, with $\lambda_{0}^{(2)}$ being the wavelength of the TEM (zero-order) mode inside the cavity. At these frequencies the zero-order mode input admittance, $Y_{0}^{\text {in, }(2)}$, is inductive and small (recall that $Y_{0}^{\mathrm{in},(2)}=0$ when the corrugation depth is an odd multiple of $\left.\lambda_{0}^{(2)} / 4\right)$. Hence, the $Y_{0}^{\text {in,(2) }}$ admittance can compensate for the small capacitive admittance introduced by evanescent harmonic/modes excited at the slit discontinuity (namely, $Y_{0}^{\mathrm{in},(2)}+\tilde{Y}^{\mathrm{eq}} \approx 0$, where $\tilde{Y}^{\mathrm{eq}}$ stands for the equivalent admittance excluding the effect of the $n=-1$ propagative harmonic). Under these circumstances, the load met at $z=0$ by the impinging signal in the circuit model is just the real admittance of the $n=-1$ propagative harmonic. If this real admittance is close to the characteristic admittance of the
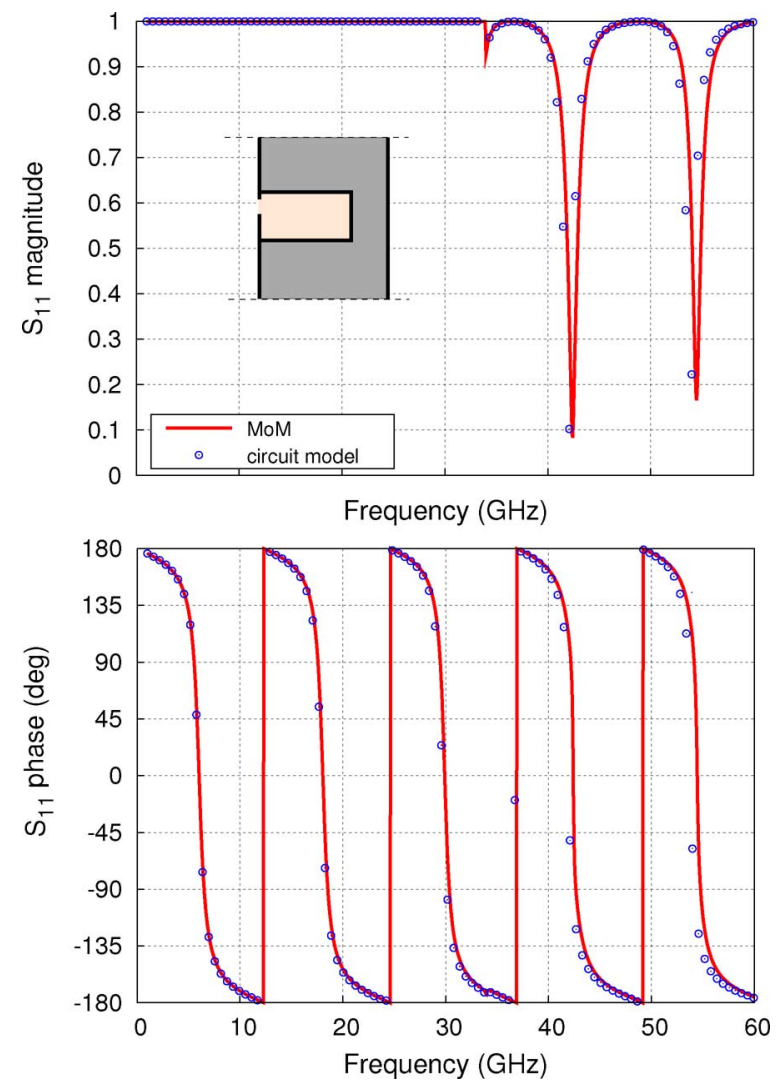

Fig. 7. Magnitude (top) and phase (bottom) of the reflection coefficient versus frequency for an structure with $a=5 \mathrm{~mm}, s=1 \mathrm{~mm}, w=0.5 \mathrm{~mm}, d_{2}=$ $5 \mathrm{~mm}, h=0.1 \mathrm{~mm}, \varepsilon_{\mathrm{r}}^{(1)}=1, \varepsilon_{\mathrm{r}}^{(2)}=6, \theta=50^{\circ}$. In our model, $N=2$, $M=1$.

input transmission line $\left(Y_{0}^{(0)}\right.$ in this case), there will be good matching and most of the impinging power is transferred to the $n=-1$ propagative harmonic (this matching is found to be better at $41.2 \mathrm{GHz}$ than at $52.7 \mathrm{GHz}$ ). At approximately $49 \mathrm{GHz}$, the whole power returns again along the input line since, at this frequency, $d_{2}=2 \lambda_{0}^{(2)}$ and then $Y_{0}^{\text {in, (2) }}=\infty$, causing a short circuit in the corresponding equivalent circuit. It should be noted that the groove size was chosen small enough to avoid the excitation of propagative modes inside the cavity in the considered frequency range $\left(s / \lambda_{0}^{(2)}<1 / 2\right)$. Thus, the present structure could be used in applications where specular reflections are not desired. Finally, it should be pointed out that the numerical results provided by our approach and the MoM agree very satisfactorily, showing a slight deviation at high frequencies as a consequence, once again, of the limitations introduced by the assumed electric field profile (see Section II-A). This satisfactory agreement has also been found for other incident angles, even for the limiting case of grazing incidence.

Previously it has been discussed that the possible application of the T-shaped corrugated structure as a high impedance surface (HIS, obtained when it behaves as an AMC) can be related to corrugation depths satisfying the condition $d_{2} \approx(2 m+$ 1) $\lambda_{0}^{(2)} / 4$. However, this HIS behavior can also be obtained in a different way by using wide and shallow grooves to make that the first propagative mode inside the cavity appears at a frequency lower than that of the first AMC condition (this situation 

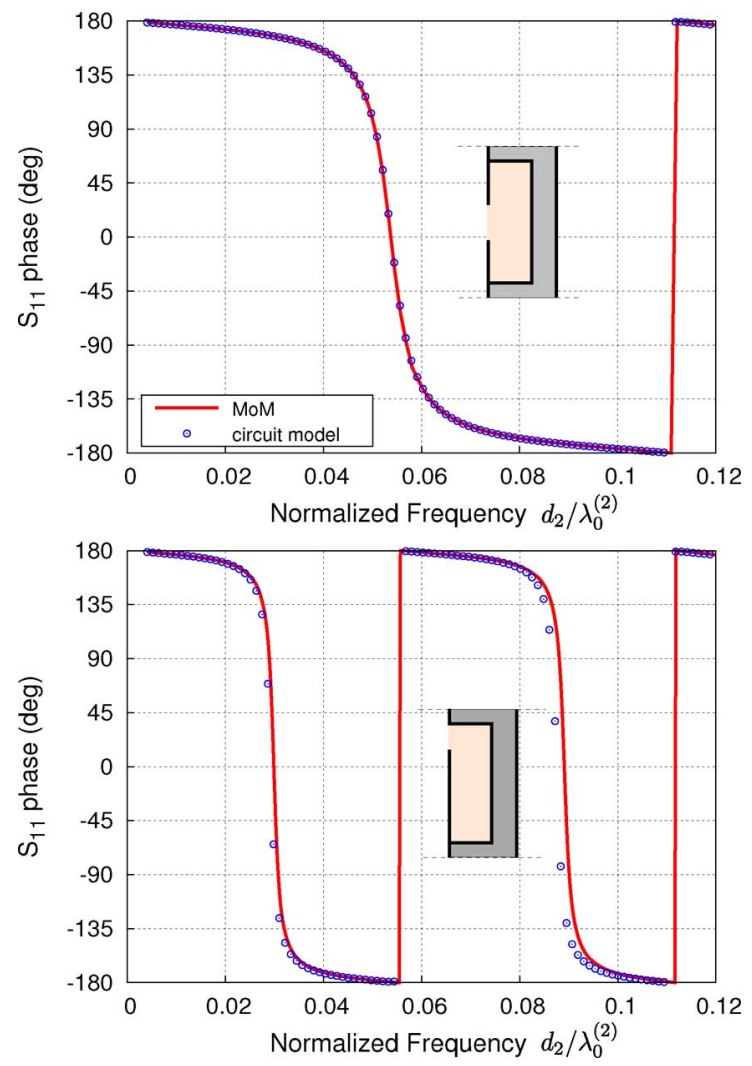

Fig. 8. Phase of reflection coefficient versus frequency for two different configurations. Top: $a=5 \mathrm{~mm}, s=4.5 \mathrm{~mm}, w=0.5 \mathrm{~mm}, d_{2}=0.5 \mathrm{~mm}$, $h=0 \mathrm{~mm}, \varepsilon_{\mathrm{r}}^{(1)}=1, \varepsilon_{\mathrm{r}}^{(2)}=5, \theta=30^{\circ}$. Bottom: Same as top, except $h=2 \mathrm{~mm} . N^{\mathrm{r}}=1, M=2$.

requires $s>2 d_{2}$ ). At this point it is convenient to recall that the modal admittances given in (16) grow to infinity as the frequency approaches any of the corresponding modal cutoff frequencies. Thus, for frequencies lower than the cutoff frequency of a given high order mode, the corresponding modal admittance is capacitive and can be as high as desired. For wide and shallow grooves, it implies that, at some frequency below the cutoff of the first high order mode inside the cavity, the capacitive admittance of the high order modes can compensate for the inductive admittance associated with the zero-order TEM standing wave inside the cavity (so that the admittance of the resulting $L C$ tank is zero). For that frequency, the corrugated structure resonates and behaves as a magnetic conductor (open circuit). This situation is reported in Fig. 8, which shows the phase of the reflection coefficient versus normalized frequency for two different configurations. In the top plot, a symmetric structure is analyzed. Since odd high order modes inside the cavity are not considered due to symmetry, the onset of the first high order mode takes place at $s / \lambda_{0}^{(2)}=1$. A near-zero phase is observed at $d_{2} / \lambda_{0}^{(2)} \approx 0.052$, corresponding to $s / \lambda_{0}^{(2)} \approx 0.466$. The bottom plot shows results obtained for an asymmetric structure with the slit fully displaced. In this case, the onset of the first odd high-order mode is half lower than that of the first even high-order mode, which causes the HIS behavior to be found also at a lower frequency $\left(d_{2} / \lambda_{0}^{(2)} \approx 0.0284\right.$, corresponding to $\left.s / \lambda_{0}^{(2)} \approx 0.255\right)$. It is interesting to note that these HIS behaviors occur when the groove size is roughly $\lambda_{0}^{(2)} / 2$ and $\lambda_{0}^{(2)} / 4$,

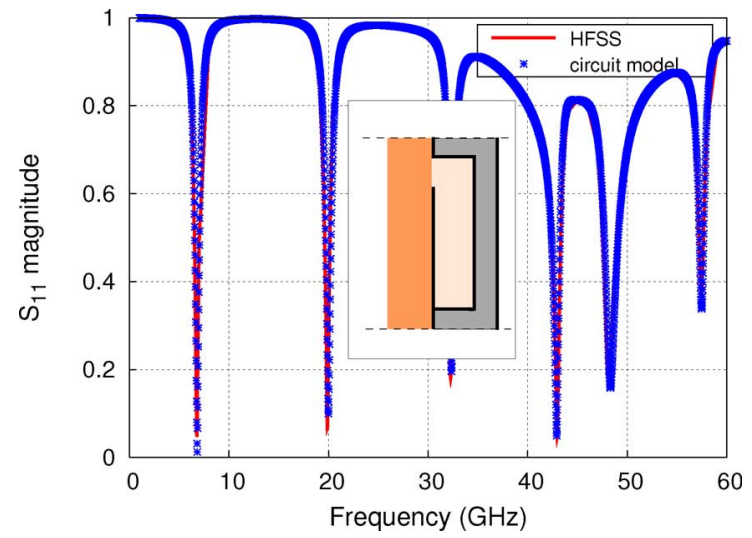

Fig. 9. Magnitude of reflection coefficient versus frequency. Structure parameters: $a=5 \mathrm{~mm}, s=4.5 \mathrm{~mm}, w=0.5 \mathrm{~mm}, d_{1}=0.5 \mathrm{~mm}, d_{2}=$ $0.5 \mathrm{~mm}, h=2 \mathrm{~mm}, \varepsilon_{\mathrm{r}}^{(0)}=1, \varepsilon_{\mathrm{r}}^{(1)}=12, \varepsilon_{\mathrm{r}}^{(2)}=5, \theta=0^{\circ}, \sigma_{1}=$ $1.088 \mathrm{~S} / \mathrm{m}, N=2, M=3$.

respectively. This is consistent with a possible alternative view [24] that considers the propagation of the TEM mode along the vertical $y$ direction inside the cavity, with the screen and the back wall now forming the parallel-plate waveguide. Under this standpoint, the structure is expected to behave as an AMC when either the upper (seen from the slit aperture) or the lower TEM line is roughly a quarter wavelength long.

The AMC behavior of the T-shaped planar corrugated structure discussed above can also be used to design a very thin narrow-band absorber. The underlying reasoning is based on the familiar idea that the electric field is maximum at the aperture under resonance condition, and therefore, it may be strongly dissipated by placing a resistive overlay with the appropriate conductivity on top of the corrugations. In principle, this appropriate value of conductivity can be found by matching the real part of the input admittance of the resistive overlay (terminated with an open circuit) to the characteristic admittance of the input transmission line, namely

$$
Y_{0}^{(0)}=\operatorname{Re}\left[Y_{0}^{\mathrm{in},(1)}\right]=\operatorname{Re}\left[\mathrm{j} Y_{0}^{(1)} \tan \left(\hat{\beta}_{n}^{(1)} d_{1}\right)\right] .
$$

Assuming that the resistive slab is electrically thin, the above condition leads to

$$
Y_{0}^{(0)} \approx \operatorname{Re}\left[\mathrm{j} \frac{\omega \varepsilon_{0} \hat{\varepsilon}_{\mathrm{r}}^{(1)}}{\hat{\beta}_{n}^{(1)}} \hat{\beta}_{n}^{(1)} d_{1}\right]=\sigma_{1} d_{1} .
$$

However, it should be noted that the condition (39) has been obtained considering only the effect that the resistive overlay has on the zeroth-order harmonic. Certainly, the higher-order harmonics will also be affected, and their corresponding admittances in the model also contribute with a real (resistive) part. In other words, the value of the overlay conductivity in (39) provides optimum absorption as long as the total field inside the resistive slab is well accounted for by the zeroth-order harmonic field, thus neglecting the strong inhomogeneity of the field in the aperture region. Nevertheless, the value of the conductivity obtained from the proposed coarse matching condition (39) can be used as an initial guess in an optimization procedure to enhance the level of absorption. Here it should be pointed out that 


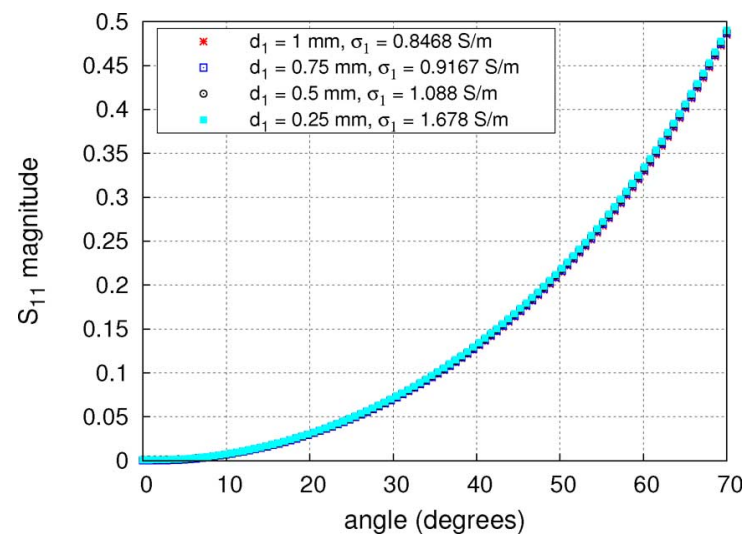

Fig. 10. Magnitude of the reflection coefficient versus incidence angle. Structure parameters: $a=5 \mathrm{~mm}, s=4.5 \mathrm{~mm}, w=0.5 \mathrm{~mm}, d_{2}=0.5 \mathrm{~mm}$, $h=2 \mathrm{~mm}, \varepsilon_{\mathrm{r}}^{(0)}=1, \varepsilon_{\mathrm{r}}^{(1)}=12, \varepsilon_{\mathrm{r}}^{(2)}=5$, Freq $=6.34 \mathrm{GHz}, N=1$, $M=1$.

the very fast numerical nature of our circuit model makes it particularly suitable for this optimization procedure. An example under normal incidence is given in Fig. 9, where the initial conductivity value given by (39) was $\sigma_{1}=5.308 \mathrm{~S} / \mathrm{m}$, and the final overlay conductivity that provides the good levels of absorption shown in the figure is $\sigma_{1}=1.088 \mathrm{~S} / \mathrm{m}$ (the relative permittivity of the cover layer is taken as $\varepsilon_{\mathrm{r}}^{(1)}=12$ to simulate a silicon substrate). Strong dissipation is observed at certain narrow frequency bands where an AMC resonance condition exists, as expected. These bands alternate with broad bands of high reflection when the frequency is far from the mentioned resonance conditions.

If the incidence angle of the impinging plane wave varies, the admittances of the lines in the model change and so does the optimal value of the conductivity. In order to study this issue, Fig. 10 shows an incidence angle scanning for different slab thicknesses at the maximum-dissipation frequency $(6.34 \mathrm{GHz})$. For each substrate thickness, the conductivity of the resistive cover layer has been optimized to obtain minimum reflection at normal incidence. It can be observed how the absorption deteriorates as the incidence angle increases. Nevertheless, the reflection coefficient magnitude is kept below $0.1(-20 \mathrm{~dB})$ for angles up to around $35^{\circ}$. Thus, for incidence between $-35^{\circ}$ and $35^{\circ}$, a reflection level below $-20 \mathrm{~dB}$ is expected. Fig. 10 also shows the remarkable fact that the angular variation of the reflection coefficient is almost independent of the overlay thickness.

As a last example, the accuracy of our equivalent circuit to model a cavity with ohmic losses is studied. In most practical cases, the cavity is usually fabricated with a good conductor and an almost negligible effect of dissipation is expected. In the present example, a lossy conductor is used in order to increase the influence of the ohmic losses. In Fig. 11 the reflection coefficient is plotted versus frequency when an oblique plane wave impinges on an asymmetric T-shaped corrugated structure. The dissipation level is low except around the frequencies for which the structure behaves as an AMC. At these particular frequencies, the reactive admittances of the corresponding equivalent circuit cancel out, resulting in a purely resistive equivalent admittance. Beyond $34 \mathrm{GHz}$, the structure works in the first grating-lobe regime and the power is split into

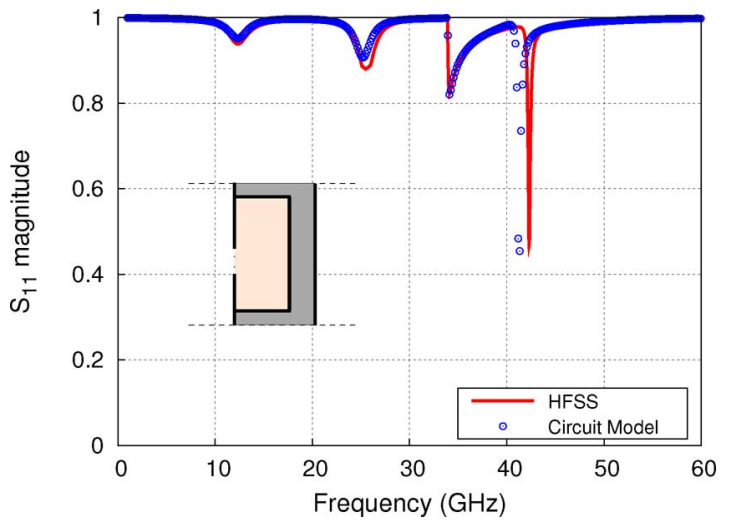

Fig. 11. Magnitude of the reflection coefficient versus normalized frequency. Structure parameters: $a=5 \mathrm{~mm}, s=3 \mathrm{~mm}, w=0.6 \mathrm{~mm}, d_{2}=1 \mathrm{~mm}$, $h=-0.6 \mathrm{~mm}, \varepsilon_{\mathrm{r}}^{(1)}=1, \varepsilon_{\mathrm{r}}^{(2)}=6, \theta=50^{\circ}, \sigma_{\mathrm{m}}=10^{6} \mathrm{~S} / \mathrm{m}, N=2$, $M=2$.

both propagative harmonics ( $n=0, n=-1)$, making the effect of losses difficult to visualize. The comparison between our model and HFSS is good for low frequencies and deteriorates at higher frequencies because of the limitations of the model.

\section{CONCLUSION}

A novel equivalent circuit approach has been presented to deal with the scattering of a TM-polarized plane wave obliquely incident on a 1-D periodic T-shaped corrugated surface with an overlay. The key features of our approach are that the topology of the circuit is deduced rigorously from a fundamental integral equation formulation and that closed-form expressions are given for all the circuit parameters. This last feature makes that the computational cost required by the present approach is very low, which can be very advantageous for design and/or optimization purposes. The numerical results obtained with the present circuit model have been compared with a rigorous fullwave MoM methods and with the HFSS simulator, as well as with other circuit approaches previously reported in the literature. The agreement is quite good with MoM and HFSS for relatively narrow slits even well beyond the diffraction regime, deteriorating slightly as the electrical size of the corrugation aperture increases. An additional advantage of our approach comes from the fact that the equivalent circuit allows for a straightforward understanding of the underlying physics of some relevant phenomena in terms of circuital and transmission line concepts. This provides us with the capability of predicting new interesting configurations with potential practical applications. Some of the possible applications that have been discussed in this work are an electrically thin HIS, a structure that avoids specular reflection, and a very thin narrow-band absorber. The equivalent circuit approach has also been extended to incorporate the effect of ohmic losses in the cavity walls.

\section{REFERENCES}

[1] R. Collin, Field Theory of Guided Waves, 2nd ed. New York, NY, USA: IEEE, 1991.

[2] D. M. Pozar, Microwave Engineering, third ed. New York, NY, USA: Wiley, 2005

[3] W. Rotman, "A study of single-surface corrugated guides," Proc. IRE, vol. 39, pp. 952-959, Aug. 1951 
[4] L. O. Goldstone and A. A. Oliner, "A note on surface waves along corrugated structures," IRE Trans. Antennas Propag., vol. 7, no. 3, pp. 274-276, 1959.

[5] G. Piefke, "The transmission characteristics of a corrugated guide," IRE Trans. Antennas Propag., vol. 7, no. 5, pp. S183-S190, 1959.

[6] H. E. M. Barlow and A. E. Karbowiak, "An experimental investigation of the properties of corrugated cylindrical surface waveguides," in Proc. IEE Part III: Radio Commun. Eng., 1954, vol. 101, pp. 182-188, 71

[7] P. S. Kildal, "Definitions of artificially soft and hard surfaces for electromagnetic waves," Electron. Lett., vol. 24, no. 3, pp. 168-170, Feb. 1988.

[8] R. S. Elliott, "On the theory of corrugated plane surfaces," IRE Trans. Antennas Propag., vol. 2, pp. 71-81, Jan. 1954.

[9] P. S. Kildal, "Artificially soft and hard surfaces in electromagnetics," IEEE Trans. Antennas Propag., vol. 38, no. 3, pp. 1537-1544, Oct. 1990.

[10] T. W. Ebbesen, H. J. Lezec, H. F. Ghaemi, T. Thio, and P. A. Wolff, "Extraordinary optical transmission through sub-wavelength hole arrays," Nature, vol. 391, pp. 667-669, Feb. 1998.

[11] F. J. García-de-Abajo, "Colloquium: Light scattering by particle and hole arrays," Rev. Modern Phys., vol. 79, pp. 1267-1290, Oct.-Dec. 2007.

[12] F. J. García-Vidal, L. Martín-Moreno, T. W. Ebbesen, and L. Kuipers, "Light passing through subwavelength apertures," Rev. Mod. Phys., vol. 82, pp. 729-787, Jan.-Mar. 2010.

[13] J. A. Porto, F. J. García-Vidal, and J. B. Pendry, "Transmission resonances on metallic gratings with very narrow slits," Phys. Rev. Lett., vol. 83, no. 14, pp. 2845-2848, Oct. 1999.

[14] T. López-Rios, D. Mendoza, F. J. García-Vidal, J. Sánchez-Dehesa, and B. Pannetier, "Surface shape resonances in lamellar metallic gratings," Phys. Rev. Lett., vol. 81, no. 3, pp. 665-668, Jul. 1998.

[15] H. E. Went, A. P. Hibbins, J. R. Sambles, C. R. Lawrence, and A. P. Crick, "Selective transmission through very deep zero-order metallic gratings at microwave frequencies," Appl. Phys. Lett., vol. 77, no. 18, pp. 2789-2791, Oct. 2000.

[16] A. N. Fantino, S. I. Grosz, and D. C. Skigin, "Resonant effects in periodic gratings comprising a finite number of grooves in each period," Phys. Rev. E, vol. 64, p. 016605, Jul. 2001.

[17] H. Shen and B. Maes, "Enhanced optical transmission through tapered metallic gratings," Appl. Phys. Lett., vol. 100, p. 241104, Jun. 2012.

[18] M. Edalatipour, A. Khavasi, M. Rezaei, and K. Mehrany, "Creation of artificial surface conductivity on metallic metamaterials," J. Lightw. Tech., vol. 30, no. 12, pp. 1789-1794, Jun. 2012.

[19] J. W. Yoon, M. J. Jung, S. H. Song, and R. Magnusson, "Analytic theory of the resonance properties of metallic nanoslit arrays," IEEE J. Quant. Electron., vol. 48, no. 7, pp. 852-861, Jul. 2012.

[20] I. M. Mandel, A. B. Golovin, and D. T. Crouse, "Analytical description of the dispersion relation for phase resonances in compound transmission gratings," Phys. Rev. A, vol. 87, p. 053833, May 2013.

[21] A. F. Harvey, "Periodic and guiding structures at microwave frequencies," IRE Trans. Microw. Theory Tech., vol. 44, no. 11, pp. 1474-1981, Nov. 1996.

[22] D. Sievenpiper, L. Zhang, R. F. Jimenez-Broas, N. G. Alexópolous, and E. Yablonovitch, "High-impedances electromagnetic surfaces with a forbidden frequency band," IEEE Trans. Microw. Theory Tech., vol. 47, no. 11, pp. 2059-2074, Nov. 1999.

[23] Z. Ying, P. S. Kildal, and A. A. Kishk, "Study of different realizations and calculation models for slot surfaces by using a vertical monopole on a soft disk as a test bed," IEEE Trans. Antennas Propag., vol. 44 no. 11 , pp. 1474-1481, Nov. 1996.

[24] E. Rajo-Iglesias, O. Quevedo, and L. Inclán-Sánchez, "Planar soft surfaces and their application to mutual coupling reduction," IEEE Trans. Antennas Propag., vol. 57, no. 12, pp. 3852-3859, Dec. 2009.

[25] E. Rajo-Iglesias, M. Caiazzo, L. Inclán-Sánchez, and P. S. Kildal, "Comparisons of bandgaps of mushroom-type EGB surface and corrugated and strip-type soft surfaces," IET Microw., Antennas Propag., vol. 1, pp. 184-189, 2007.

[26] E. Alfonso, A. Valero-Nogueira, J. I. Herranz, and F. Vico, "Moment method analysis of corrugated surfaces using the aperture integral equation," IEEE Trans. Antennas Propagat., vol. 57, no. 7, pp. 2208-2212, Jul. 2009.

[27] N. N. M. Kehn, "Moment method analysis of plane-wave scattering from planar corrugated surfaces using parallel-plate cavity Green's function and derivation of analytic reflection-phase formulas for both polarizations and oblique azimut planes," Radio Sci., vol. 47, p. RS3008, Jun. 2012.

[28] N. N. M. Kehn, "Rapid surface-wave dispersion and plane-wave reflection analyses of planar corrugated surfaces by asymptotic corrugations boundary conditions even for oblique azimuth planes," IEEE Trans. Antennas Propag., vol. 61, no. 5, pp. 2695-2707, May 2013.
[29] D. W. Woo, S. J. Muhn, and W. S. Park, "Simple analytical model of propagation through thick periodic slots," IEEE Trans. Antennas Propag., vol. 60, no. 11, pp. 5329-5335, Nov. 2012.

[30] F. Medina, F. Mesa, and R. Marqués, "Extraordinary transmission through arrays of electrically small holes from a circuit theory perspective," IEEE Trans. Microw. Theory Tech., vol. 12, no. 12, pp. 3108-3120, Dec. 2008.

[31] R. Rodríguez-Berral, F. Medina, and F. Mesa, "Circuit model for a periodic array of slits sandwiched between two dielectric slabs," Appl. Phys. Lett., vol. 96, pp. 161104-161104, Apr. 2010.

[32] R. Rodríguez-Berral, C. Molero, F. Medina, and F. Mesa "Analytical wideband model for strip/slit gratings loaded with dielectric slabs," IEEE Trans. Microw. Theory Tech., vol. 60, no. 12, pp. 3908-3918, Dec. 2012

[33] ANSYS High Frequency Structure Simulator (HFSS), [Online]. Available: http://www.ansys.com/Products/Simulation+Technology/Electromagnetics/High-Performance+Electronic+Design/ANSYS+HFSS

[34] F. Medina, F. Mesa, and D. C. Skigin, "Extraordinary transmission through arrays of slits: A circuit theory model," IEEE Trans. Microw. Theory Tech., vol. 58, no. 1, pp. 105-115, Jan. 2010.

[35] R. Yang, R. Rodríguez-Berral, F. Medina, and Y. Hao, "Analytical model for the transmission of electromagnetic waves through arrays of slits in perfect conductors and lossy metal screens," J. Appl. Phys., vol. 109, p. 103107, May 2011.

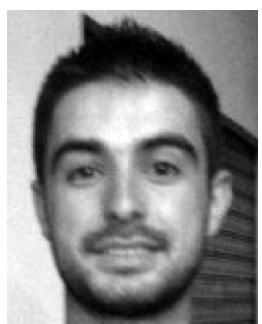

Carlos Molero was born in Las Navas, Seville, Spain, in 1987. He received the Licenciado degree in Physics from the Universidad de Sevilla, Seville, Spain, in 2011, where he is currently pursuing the Ph.D. degree in the Department of Applied Physics 1.

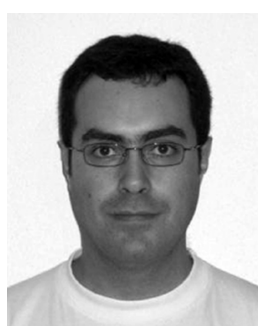

Raúl Rodríguez-Berral was born in Casariche, Seville, Spain, in 1978. He received the Licenciado (M.Sc.) and Ph.D. degrees in physics from the Universidad de Sevilla, Seville, Spain, in 2001 and 2008, respectively.

In January 2002, he joined the Department of Applied Physics 1, Universidad de Sevilla, where he is currently an Assistant Professor. His research interests include the study of the spectrum and the excitation of periodic and nonperiodic planar structures and high-frequency circuit modeling.

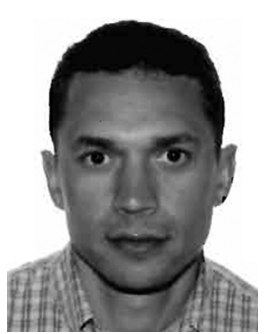

Francisco Mesa (M'93-SM'11-F'14) was born in Cádiz, Spain, in April 1965. He received the Licenciado and Doctor degrees in physics from the Universidad de Sevilla, Seville, Spain, in 1989 and 1991, respectively.

He is currently Professor in the Departamento de Física Aplicada 1, Universidad de Sevilla. His research interests focus on electromagnetic propagation/radiation in planar structures.

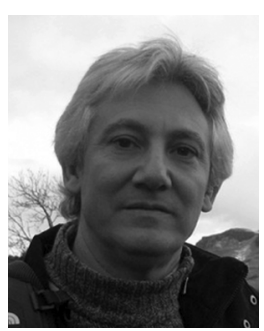

Francisco Medina (M'90-SM'01-F'10) was born in Puerto Real, Cádiz, Spain, in November 1960. He received the Licenciado and Doctor degrees from the Universidad de Sevilla, Seville, Spain, in 1983 and 1987, respectively, both in physics.

$\mathrm{He}$ is currently Professor of electromagnetism in the Department of Electronics and Electromagnetism, University of Seville, and Head of the Microwaves Group. His research interest includes analytical and numerical methods for planar structures, anisotropic materials, and artificial media modeling. He has coauthored about 120 journal papers on those topics. 